\title{
A multifactorial regression analysis of the features of community-acquired rotavirus requiring hospitalization in Spain as represented in the Minimum Basic Data Set
}

\author{
O. REDONDO-GONZÁLEZ ${ }^{1,2 *}$ AND J. M. TENÍAS-BURILLO ${ }^{3}$ \\ ${ }^{1}$ Research Support Unit, La Mancha Centro General Hospital. Alcázar de San Juan, Ciudad Real, Spain \\ ${ }^{2}$ Social and Cardiovascular Epidemiology Research Group, School of Medicine, University of Alcalá, Alcalá de \\ Henares, Madrid, Spain \\ ${ }^{3}$ Preventive Medicine Unit, Pare Jofré Hospital, Valencia, Spain
}

Received 22 December 2015; Final revision 1 April 2016; Accepted 14 April 2016; first published online 6 May 2016

\section{SUMMARY}

Over $10 \%$ of acute rotavirus gastroenteritis (ARGE) requires hospitalization because of complications. The aggravating factors have been widely analysed, but in an isolated way. We aimed to explore the interrelationship between the clinical and epidemiological factors that characterize rotavirus hospitalizations in Spain using information from the Minimum Basic Data Set (MBDS). Using ICD-9-CM codes, we classified acute gastroenteritis (AGE) cases by principal diagnosis fields and then categorized their comorbidities, complications, and epidemiological features by secondary fields. A multivariable, logistic, step-wise regression model was then constructed. We identified 1657 ARGE cases from 17415 cases of AGE. Rotavirus hospitalizations were associated with place of residence, age, and season $(P<0 \cdot 0001)$, as well as with dehydration [odds ratio (OR) 12.44, 95\% confidence interval (CI) 1.52-40.38], intravenous rehydration (OR 1.74, 95\% CI 1·29-2·35), metabolic acidosis (OR 1.51, 95\% CI 1·24-1·83), respiratory tract infections (RTIs) (OR 1·60, 95\% CI 1.09-1.98), and concomitant AGE (OR $1 \cdot 52,95 \%$ CI $1 \cdot 03-2 \cdot 25$ ). Dehydration was four times more likely in patients aged $<5$ years (OR 4.36, 95\% CI 1.20-12.96) and was associated with acidosis when ARGE and RTI were present simultaneously $(P<0 \cdot 0001)$. Specific co-infecting viruses may play a role in acute respiratory symptoms and aggravation of gastrointestinal manifestations of rotaviruses, thus leading to complications requiring hospitalization.

Key words: Clinical features, epidemiology, medical registries, rotavirus, Spain.

\section{INTRODUCTION}

Acute rotavirus gastroenteritis (ARGE) is characterized by a more severe clinical course and a higher number of potential complications than other types of infectious acute gastroenteritis (AGE), and is observed predominantly in children aged $<2$ years

\footnotetext{
* Author for correspondence: O. Redondo-González, MD, MPH, Avda. Constitución 3, Alcázar de San Juan, 13600 Ciudad Real, Spain.

(Email: oredgon@gmail.com)
}

$[1,2]$. Mild fever occurs in $65 \cdot 5 \%$, severe dehydration in $49 \cdot 9 \%$, and metabolic acidosis in $79 \cdot 2 \%$ of cases [3], with the dehydration rate being $32 \%$ higher for rotavirus [2]. Vomiting, diarrhoea, or severe febrile ARGE can lead to moderate to severe dehydration requiring acute rehydration in hospital [3]. In the developed world, complications from ARGE requiring hospitalization occur in $>10 \%$ of children, with hypertonic dehydration $(5 \cdot 3 \%)$ and seizures $(1 \cdot 7 \%)$ being the most frequent reasons for hospital admission [4]. Aggravating factors affect the severity of the 
condition, with rates of hospitalization differing between countries and regions in Europe [3].

The isolated influence of perinatal factors, as well as the demographic (maternal and family issues) and clinical aspects of this type of hospitalization have all been reported [1, 5-8]. To date, the known characteristics of rotavirus that lead to a predisposition to hospitalization include: congenital malformations, low birth weight (LBW), male gender, maternal smoking, single mother, daycare for children aged $<24$ months, siblings aged $<1$ year at home, maternal age $<25$ years, low level of maternal education $(<$ secondary school), and lack of breastfeeding [8, 9]. Furthermore, $41.2 \%$ of ARGE cases have been associated with acute respiratory tract infections (RTIs) [10], with respiratory signs appearing in $60.7 \%$ of ARGE cases [11]. However, the role of common respiratory viruses in enteric infections has yet to be elucidated $[12,13]$. Human coronaviruses were detected in $6.4 \%$ of children with AGE and in $7 \cdot 1 \%$ of those with symptoms of both AGE and RTI [12]. Additionally, $83 \%$ of ARGE cases were found to be comorbid with human bocaviruses in children aged $<4$ years [13, 14]. Rotavirus hospitalizations are also more prevalent in children with dystrophy, irondeficiency anaemia, intestinal parasitosis, rhinopharyngitis, or pneumococcal meningitis. In fact, symptoms of upper RTI, acute pharyngitis, and maculopapular eruption accompany ARGE in 13.3\%, 10\% and $16 \cdot 6 \%$ of cases, respectively [15]. However, until now we have found no studies evaluating the confluence of comorbidities, clinical course, and epidemiology of ARGE cases requiring hospitalization within the same cohort.

The aim of this study is to explore the interrelationship of clinical and epidemiological factors associated with community-acquired rotavirus requiring hospitalization in Spain.

\section{METHODS}

\section{Design and setting}

This retrospective cohort study was performed as part of a larger project carried out in Castile-La Mancha (CLM), an autonomous region in central Spain, in order to describe the burden borne by hospitals due to community-acquired rotavirus both before and after a 3-year period in which rotavirus vaccines were made available in Spain. Data were obtained for all AGE-associated hospitalizations in CLM from 1 January 2003 to 31 December 2009. Two data sources were used: the Minimum Basic Data Set (MBDS) of the CLM Health Service and the Statistics on Health Establishments with an Inpatient Regimen, both of which represent a population coverage of $99 \cdot 37 \%$.

\section{Definition of the variable of interest}

A total of 23006 hospital discharges with any records that included a primary or secondary diagnosis of AGE were retrieved from the MBDS. According to the Ninth Revision of the International Classification of Diseases, Clinical Modification (ICD-9-CM), AGE cases are classified as either bacterial or parasitic (001-005, 006-007, 008.0-008·5), viral (008.6-008.8), 'of undetermined cause' (009.0-009.3), 'unspecified non-infectious AGE' (558.9), or 'diarrhoea' (787.91).

Community-acquired rotaviruses were defined as having the ICD-9-CM code 008.61 either in the primary diagnosis field of the MBDS or in one of the secondary diagnosis fields (SDFs) together with a principal diagnosis code of 'undetermined aetiology', 'cause-unspecified', or 'diarrhoea'.

Other possible cases of community-acquired AGE were identified from records that had the ICD-9-CM bacterial, parasitic, or viral AGE codes, as specified above, in the primary diagnosis field, as well as those records that contained these codes in a SDF together with a principal diagnosis of 'undetermined aetiology', 'cause-unspecified', or 'diarrhoea'. Concomitant AGE cases were defined as having any of the bacterial, parasitic, or viral AGE codes in the SDF. The algorithm explaining the final count has been described elsewhere [16].

\section{Definition of the independent (exposure) variables}

ICD-9-CM codes in the SDFs of whatever AGE cases identified were revised, from the first to the thirteenth, to assess the features of all AGE hospitalizations:

\section{Complications}

- Dehydration: $276 \cdot 5$ (low volume), $276 \cdot 50$ (unspecified volume decrease), 276.51 (dehydration), 276.52 (hypovolaemia), 276.0 (hypernatraemic), $276 \cdot 1$ (hyponatraemic).

- Acidosis: $276 \cdot 2$ (unspecified, respiratory, lactic or metabolic); not diabetic.

- Hypoglycaemia: $251 \cdot 2$ (unspecified). 
- Seizures: 779.0 (newborn seizures), 780·31 (unspecified simple febrile seizures), $780 \cdot 32$ (complex febrile seizures), and 780.39 (others: unspecified attacks, recurrent seizures or syncope; seizures not otherwise specified).

- Intravenous (IV) rehydration: 39.96 in any of the first to eighth 'surgical and/or obstetric procedures' fields of the MBDS.

\section{Comorbidities}

- Perinatal problems (PNPs): V13.7 (perinatal problems), V21.3 (state of LBW: V21·30-V21·35), and 760-779 (diseases in the perinatal period).

- Disorders of pondostatural development (DPSD): 783.0 (anorexia), 783.4 (lack of expected normal physiological development), 783.40 (unspecified lack of normal physiological development), 783.41 (inadequate weight gain), 783.43 (short stature), 263.9 (unspecified protein-calorie malnutrition), 783.2 (abnormal weight loss), 783.21 (weight loss), and 783.22 (underweight).

- Malabsorption syndrome: 579.8 (specified intestinal absorption defect), 579.0 (celiac disease), and 579.9 (unspecified intestinal malabsorption).

- Gastroesophageal reflux disease (GERD): 530·81.

- Atopic dermatitis and related conditions: 691.0 (diaper rash), 691.8 [atopic, flexural, or intrinsic (allergic) eczema; atopic or diffuse (Brocq) neurodermatitis; Besnier's prurigo].

- Iron-deficiency anaemia: 280.9.

- RTI. Upper RTI: 460 (nasopharyngitis/common cold), 462 (pharyngitis), 463 (tonsillitis), 464.0 (laryngitis: $464 \cdot 00$ and $464 \cdot 01$, without/with obstruction), 464.1 (tracheitis: 464.10 and 464.11), 464 (unobstructed laryngitis), $465 \cdot 8$ (upper RTI of multiple locations), 465.9 (upper RTI of unspecified location). Otitis: $381 \cdot 00$ [unspecified non-suppurative otitis media (OM)], 381.01 (serous OM), 381.4 (non-suppurative OM, not classified as acute or chronic), 382.00 and 382.01 (suppurative OM without/with ruptured eardrum), $382 \cdot 4$ (unspecified suppurative OM), 382.9 (unspecified OM). Lower acute RTI: $466 \cdot 0$ (bronchitis), 466.11 (respiratory syncytial virus bronchiolitis), $466 \cdot 19$ (bronchiolitis due to other agents), $480 \cdot 9$ (unspecified viral pneumonia), 481 (pneumococcal pneumonia), 485 and 486 (unspecified bronchopneumonia).

- Urinary tract infection (UTI): 599.0.

- Concomitant gastrointestinal infection: Salmonella (003·0, 003·1, 003·8, 003.9), Pseudomonas (008·42),
Campylobacter (008.43), Clostridium difficile (008.45), adenovirus enteritis (008.62), other viral enteritis (toroviruses) (008.69).

- Oral candidiasis: $112 \cdot 0$.

\section{Epidemiological features}

- Sex and age groups (<7 and 7-11 months; 1, 2, 3, $4,5-9,10-14$ and $\geqslant 15$ years).

- Hospitalization period: 2003-2005 (prior to vaccine availability) vs. 2007-2009 (3-year period when Rotarix (GlaxoSmithKline, Spain) and RotaTeq (Sanofi Pasteur MSD, Spain) vaccines became commercially available simultaneously in Spain).

- Season of hospitalization: December-February, March-May, June-August, September-November.

\section{Statistical analysis}

First, we calculated both the number of cases and the percentages of the codes most frequently noted in the hospital discharge information for cause-specific infectious (known) AGE, as well as for AGE of unknown aetiology.

The frequency of all independent variables or features was described both in the group of patients hospitalized for ARGE, as well as in the group of those hospitalized for other AGE. A simple (bivariate) logistic regression analysis was performed to compare the occurrence of each feature in these groups. The odds ratio (OR) and 95\% confidence interval (CI) showed the effect size of each independent variable in the ARGE group relative to that in the AGE group. A $\chi^{2}$ test was used to explore the association between dehydration and other characteristics of the ARGE cases identified.

To analyse the associations between communityacquired ARGE requiring hospitalization and the epidemiological and clinical factors described above (both complications and comorbidities), we constructed a multivariable, logistic, step-wise regression model by introducing the variables that were found to be significant in the bivariate analysis at each step in the block for their entry or removal.

All analyses were performed with Stata v. 11 statistical software (StataCorp LP, USA); the level of significance $(P)$ was assigned to values $<0 \cdot 05$.

\section{Ethics}

The Ethical Review Board of The Service of Information and Sanitary Statistics of the Counselling of Health and Social Welfare in CLM approved this study prior to establishing a Firm Commitment 
Table 1. Number of discharges of general AGE cases, by age group (source: MBDS, CLM, Spain, 2003-2009)

\begin{tabular}{|c|c|c|c|c|c|c|c|}
\hline Age & $\begin{array}{l}\text { Rotavirus, } \\
\%(N)\end{array}$ & $\begin{array}{l}\text { Salm. spp.*, } \\
\%(N)\end{array}$ & $C j, \%(N)$ & $\begin{array}{l}\text { Other known } \dagger \\
\text { AGE, } \%(N)\end{array}$ & $\begin{array}{l}\text { Unknown } \\
\text { AGE, } \%(N)\end{array}$ & $\begin{array}{l}\text { Overall AGE, } \\
\%(N)\end{array}$ & $\begin{array}{l}\% \text { rotavirus/ } \\
\text { overall } \mathrm{AGE}\end{array}$ \\
\hline$<7$ mo. & $24 \cdot 3(403)$ & $3 \cdot 4(53)$ & $22 \cdot 3(75)$ & $9 \cdot 3(134)$ & $7 \cdot 6(938)$ & $9 \cdot 2(1603)$ & $25 \cdot 1$ \\
\hline 7-11 mo. & $25 \cdot 7(426)$ & $2 \cdot 2(35)$ & $9 \cdot 2(31)$ & $6 \cdot 2(89)$ & $1 \cdot 1(140)$ & $4 \cdot 1(721)$ & $59 \cdot 1$ \\
\hline $1 \mathrm{yr}$ & $33 \cdot 8(561)$ & $5 \cdot 9(94)$ & $12 \cdot 2(41)$ & $8 \cdot 2(118)$ & $8 \cdot 9(1104)$ & $11 \cdot 0(1918)$ & $29 \cdot 2$ \\
\hline $2 \mathrm{yr}$ & $9 \cdot 3(154)$ & $5 \cdot 9(94)$ & $7 \cdot 4(25)$ & $4 \cdot 0(58)$ & $4 \cdot 5(559)$ & $5 \cdot 1(890)$ & $17 \cdot 3$ \\
\hline $3 \mathrm{yr}$ & $3 \cdot 1(51)$ & $4 \cdot 2(67)$ & $3 \cdot 6(12)$ & $2 \cdot 1(30)$ & $2 \cdot 9(358)$ & $3 \cdot 0(518)$ & $9 \cdot 8$ \\
\hline $4 \mathrm{yr}$ & $1 \cdot 7(28)$ & $3 \cdot 8(61)$ & $4 \cdot 7(15)$ & $1 \cdot 9(27)$ & $2 \cdot 1(256)$ & $2 \cdot 2(387)$ & $7 \cdot 2$ \\
\hline$<5 \mathrm{yr}$ & $97 \cdot 9(1623)$ & $25 \cdot 5(404)$ & $59 \cdot 3(199)$ & $31 \cdot 8(456)$ & $27(3355)$ & $34 \cdot 7(6037)$ & $26 \cdot 9$ \\
\hline $5-9 \mathrm{yr}$ & $1 \cdot 5(25)$ & $12 \cdot 8(203)$ & $10 \cdot 4(35)$ & $5 \cdot 8(83)$ & $6 \cdot 4(795)$ & $6 \cdot 6(1141)$ & $2 \cdot 2$ \\
\hline $10-14 \mathrm{yr}$ & $0 \cdot 2(4)$ & $6 \cdot 2(98)$ & $2 \cdot 1(7)$ & $2 \cdot 6(37)$ & $2 \cdot 9(361)$ & $2 \cdot 9(507)$ & $0 \cdot 8$ \\
\hline$\geqslant 14 \mathrm{yr}$ & $0 \cdot 3(5)$ & $55 \cdot 5(877)$ & $28 \cdot 2(95)$ & $59 \cdot 8(860)$ & $63 \cdot 6(7893)$ & $55 \cdot 9(9730)$ & $0 \cdot 1$ \\
\hline Total & $100(1657)$ & $100(1581)$ & $100(337)$ & $100(1437)$ & $100(12403)$ & $100(17415)$ & $9 \cdot 5$ \\
\hline
\end{tabular}

AGE, Acute rotavirus gastroenteritis; MBDS, Minimum Basic Data Set; CLM, Castile-La Mancha; Cj, Campylobacter jejuni.

* Diagnoses of localized Salmonella infection (no AGE) $(003 \cdot 21-003 \cdot 24)$ were excluded.

$\dagger$ Rest of AGE cases defined by a specific code in the primary diagnosis field of MBDS, together with AGE cases coded as AGE 'of undetermined aetiology' (009·0-009·3), 'Unspecified non-infectious AGE' (558·9), or 'Diarrhoea' (787.91), with a specifically coded AGE diagnosis in any secondary field of the MBDS.

† Unknown AGE, AGE coded as AGE 'of undetermined aetiology' (009·0-009·3), 'Unspecified non-infectious AGE' (558·9), or 'Diarrhoea' (787.91), without a specifically coded AGE diagnosis in any secondary field of the MBDS.

Requirement of a Confidentiality Agreement, as set forth by Royal Decree 994/1999, which regulates the security measures for automated files containing personal data.

\section{RESULTS}

We identified 17415 AGE cases from 22998 registries, of which 1657 were identified as ARGE (99.7\% were emergency admissions). The median number of rotavirus admissions per year was 203 [interquartile range (IQR) 190-263], accounting for $10 \%$ of discharges with a classification of AGE throughout the study period $(27 \%$ in children aged $<5$ years) (Table 1$)$. The second and third most common cause-specific AGE in children aged $<5$ years were Salmonella and Campylobacter, which made up $7 \%$ and $3 \%$ of all AGE cases, respectively, in this age group (Table 1). Half of all rotavirus hospitalizations occurred in children aged $<2$ years, with a median age of 1 year (IQR $0 \cdot 59-1 \cdot 63$ ); $56 \%$ were male (Table 2 ). In contrast, $57 \%$ of admissions for other AGE occurred in children aged $\geqslant 10$ years, and only $14 \%$ appeared in children aged $<1$ year (median age 32·3, IQR 2·4-73·2) (Table 2).

Eighteen percent of patients with ARGE had one co-infection $(P=0.027)$; only $0.1 \%$ presented with two co-infections. Of all rotaviruses complicated with dehydration, $14 \%$ had one or more co-infections. Frequent co-infecting rotavirus agents included adenovirus (70\%), followed by Campylobacter (19\%) and Salmonella (8\%). Almost $68 \%$ of rotavirus hospitalizations occurred in Ciudad Real and Guadalajara. With respect to other AGE cases, dehydration, acidosis, and hypoglycaemia complications were $12 \%$, $8 \%$, and $2 \%$ more frequent, respectively; the need for IV infusion was 3\% greater. Moreover, the occurrence of concomitant RTIs and AGE was $9 \%$ and $2 \%$ higher, respectively. Rotaviruses were also significantly more commonly associated with GERD, seizures, or PNPs, among others (Table 2).

Dehydration was four times more frequent in ARGE patients aged $<5$ years (OR $4 \cdot 36,95 \%$ CI $1 \cdot 20-12 \cdot 96, P=0 \cdot 024)$. Twenty-one percent $(48 / 232)$ of children with ARGE and a RTI $(P=0.035)$ and 9\% (3/32) of those with ARGE accompanied by GERD $(P=0 \cdot 028)$ were dehydrated. However, dehydration was not a significant complication in children with ARGE and a malabsorption disorder $(P=$ $0.695)$, nor in the case of ARGE with a concomitant AGE $(P=0.053)$. Twenty-seven percent $(118 / 436)$ of rotavirus dehydrations presented with acidosis $(P<$ $0 \cdot 0001)$. Dehydration was associated with acidosis when ARGE and RTI were simultaneously present $(P<0.0001)$, but not in the case of ARGE and a concomitant AGE $(P=0.578)$, nor in children with ARGE who also presented with malabsorption syndrome $(P=0 \cdot 512)$ or GERD $(P=0 \cdot 320)$. Neither patients with ARGE and PNPs nor ARGE and 
Table 2. Description and bivariate analysis of characteristics of patients hospitalized for rotavirus (source: MBDS, CLM, Spain, 2003-2009)

\begin{tabular}{|c|c|c|c|c|c|}
\hline Exposure variable & Rotavirus, $\%(N=1657)$ & Other AGE, $\%(N=15758)$ & OR & $95 \% \mathrm{CI}$ & $P$ \\
\hline \multicolumn{6}{|l|}{ Age } \\
\hline$<1 \mathrm{yr}$ & $50 \cdot 1(829)$ & $13 \cdot 8(2176)$ & \multicolumn{2}{|c|}{ Reference } & $*$ \\
\hline $1 \mathrm{yr}$ & $33 \cdot 8(561)$ & $10 \cdot 2(1611)$ & 0.91 & $0 \cdot 80-1 \cdot 03$ & \\
\hline $2 \mathrm{yr}$ & $9 \cdot 3(154)$ & $5 \cdot 4(845)$ & $0 \cdot 48$ & $0 \cdot 40-0 \cdot 58$ & \\
\hline $3-4 \mathrm{yr}$ & $4 \cdot 8(79)$ & $5 \cdot 9(930)$ & $0 \cdot 22$ & $0 \cdot 17-0 \cdot 28$ & \\
\hline $5-9 \mathrm{yr}$ & $1 \cdot 5(25)$ & $7 \cdot 9(1250)$ & $0 \cdot 05$ & $0 \cdot 04-0 \cdot 08$ & \\
\hline$\geqslant 10 \mathrm{yr}$ & $0.5(9)$ & $56.9(8946)$ & $0 \cdot 002$ & $0 \cdot 001-0 \cdot 005$ & \\
\hline \multicolumn{6}{|l|}{ Sex } \\
\hline Men & $56 \cdot 4(935)$ & $50 \cdot 4(7964)$ & \multicolumn{2}{|c|}{ Reference } & $*$ \\
\hline Women & $43 \cdot 6(722)$ & $49 \cdot 6(7794)$ & $0 \cdot 78$ & $0 \cdot 71-0.87$ & \\
\hline \multicolumn{6}{|l|}{ Province of residence } \\
\hline Albacete & $12 \cdot 3(204)$ & $16 \cdot 3(2576)$ & \multicolumn{2}{|c|}{ Reference } & $*$ \\
\hline Toledo & $10 \cdot 0(165)$ & $28 \cdot 7(4522)$ & $0 \cdot 60$ & $0 \cdot 48-0 \cdot 74$ & \\
\hline Cuenca & $9 \cdot 7(161)$ & $17 \cdot 9(2815)$ & $0 \cdot 86$ & $0 \cdot 70-1 \cdot 07$ & \\
\hline Guadalajara & $22 \cdot 4(375)$ & $12 \cdot 8(2024)$ & $2 \cdot 56$ & $2 \cdot 14-3 \cdot 06$ & \\
\hline Ciudad Real & $45 \cdot 4(751)$ & $24 \cdot 3(3822)$ & $2 \cdot 29$ & $1.95-2.69$ & \\
\hline \multicolumn{6}{|l|}{ Hospitalization period } \\
\hline 2003-2005 & $46 \cdot 4(769)$ & $43 \cdot 6(6870)$ & \multicolumn{2}{|c|}{ Reference } & $0 \cdot 058$ \\
\hline 2007-2009 & $39 \cdot 9(662)$ & $41 \cdot 7(6,73)$ & $0 \cdot 90$ & $0 \cdot 81-1 \cdot 00$ & \\
\hline \multicolumn{6}{|l|}{ Season } \\
\hline December-February & $57(942)$ & $25 \cdot 8(4060)$ & \multicolumn{2}{|c|}{ Reference } & $*$ \\
\hline March-May & $17(277)$ & $20 \cdot 4(3212)$ & $0 \cdot 37$ & $0 \cdot 32-0 \cdot 43$ & \\
\hline June-August & $5(88)$ & $26 \cdot 6(4193)$ & $0 \cdot 08$ & $0 \cdot 07-0 \cdot 11$ & \\
\hline September-November & $21(349)$ & $27 \cdot 2(4294)$ & $0 \cdot 35$ & $0 \cdot 31-0 \cdot 40$ & \\
\hline Dehydration & $26 \cdot 3(436)$ & $14 \cdot 3(2259)$ & $5 \cdot 40$ & $0 \cdot 73-39 \cdot 82$ & $0 \cdot 098$ \\
\hline Intravenous rehydration & $4 \cdot 5(75)$ & $1 \cdot 6(230)$ & $3 \cdot 20$ & $2 \cdot 46-4 \cdot 18$ & $*$ \\
\hline Acidosis & $12 \cdot 6(209)$ & $4 \cdot 4(693)$ & $3 \cdot 14$ & $2 \cdot 67-3 \cdot 70$ & $*$ \\
\hline Hypoglycaemia & $2 \cdot 2(36)$ & $0 \cdot 7(117)$ & $2 \cdot 97$ & $2 \cdot 04-4 \cdot 33$ & $*$ \\
\hline Respiratory tract infection & $14(232)$ & $4 \cdot 7(736)$ & $3 \cdot 33$ & $2 \cdot 84-3 \cdot 89$ & $*$ \\
\hline Concomitant AGE & $2 \cdot 4(39)$ & $0 \cdot 4(56)$ & $6 \cdot 76$ & $4 \cdot 48-10 \cdot 21$ & $*$ \\
\hline Urinary tract infection & $1 \cdot 3(21)$ & $2 \cdot 8(447)$ & $0 \cdot 44$ & $0 \cdot 28-0.68$ & $*$ \\
\hline GERD & $1 \cdot 9(32)$ & $1(148)$ & $2 \cdot 08$ & $1 \cdot 41-3 \cdot 06$ & $*$ \\
\hline Seizures & $1(16)$ & $0 \cdot 3(42)$ & $3 \cdot 65$ & $2 \cdot 05-6 \cdot 51$ & $*$ \\
\hline DPSD & $3 \cdot 5(58)$ & $2 \cdot 0(315)$ & $1 \cdot 78$ & $1 \cdot 34-2 \cdot 37$ & $0 \cdot 001$ \\
\hline PNPs & $2 \cdot 1(34)$ & $1 \cdot 2(86)$ & $3 \cdot 82$ & $2 \cdot 56-5 \cdot 70$ & $*$ \\
\hline Malabsorption disorder & $1 \cdot 6(27)$ & $0 \cdot 5(146)$ & $1 \cdot 77$ & $1 \cdot 17-2 \cdot 68$ & 0.007 \\
\hline Iron-deficiency anaemia & $2 \cdot 1(34)$ & $2 \cdot 3(364)$ & $0 \cdot 89$ & $0.62-1 \cdot 26$ & $0 \cdot 506$ \\
\hline Oral candidiasis & $0 \cdot 8(13)$ & $0.5(79)$ & $1 \cdot 57$ & $0 \cdot 87-2,83$ & $0 \cdot 133$ \\
\hline Atopic dermatitis & $1 \cdot 7(28)$ & $0 \cdot 6(95)$ & $2 \cdot 84$ & $1 \cdot 85-4 \cdot 34$ & $*$ \\
\hline
\end{tabular}

MBDS, Minimum Basic Data Set; CLM, Castile-La Mancha; AGE, acute gastroenteritis; OR, Odds ratio; CI, confidence Interval; GERD, gastroesophageal reflux disease; DPSD, disorders of pondostatural development; PNPs, perinatal problems. $* P<0.0001$.

DPSD showed a greater risk of dehydration $(P=$ $0 \cdot 708$ and $P=0 \cdot 258$, respectively).

In the multivariate analysis, the characteristics that were found to be independently associated with rotavirus hospitalizations were age, province, season of hospitalization, acidosis (all $P<0 \cdot 0001$ ), dehydration $(P=0.0003)$, IV rehydration $(P=0.0020)$, RTI $(P=$ $0.0033)$, and concomitant AGE $(P=0.0355)$ (Table 3). At age 2 years, the risk of rotavirus hospitalization decreased to less than half and between 3 and 4 years, to a quarter. In summer, admissions were reduced by $92 \%(95 \%$ CI $0 \cdot 05-0 \cdot 14)$. Compared to other AGE cases, dehydration was 12 times more likely in ARGE cases (95\% CI 1.52-40.38) (Table 3), while acidosis (95\% CI 1.24-1.83), the need for IV infusion (95\% CI $1 \cdot 29-2 \cdot 35)$, and the presence of a concomitant AGE $(95 \%$ CI $1 \cdot 03-2 \cdot 25)$ or RTI (95\% CI 1.09-1.98) were almost double. 
Table 3. Multivariate analysis of those characteristics associated with rotavirus hospitalizations in the bivariate analysis (source: MBDS, CLM, Spain, 20032009)

\begin{tabular}{lrll}
\hline \hline Exposure variable & OR & $95 \%$ CI & $P$ \\
\hline Age & & & \\
1 yr & $0 \cdot 73$ & $0 \cdot 56-0 \cdot 98$ & $*$ \\
2 yr & $0 \cdot 41$ & $0 \cdot 27-0 \cdot 61$ & \\
$3-4 \mathrm{yr}$ & $0 \cdot 21$ & $0 \cdot 12-0 \cdot 35$ & \\
$5-9 \mathrm{yr}$ & $0 \cdot 02$ & $0 \cdot 005-0 \cdot 08$ & \\
$\geqslant 10 \mathrm{yr}$ & $0 \cdot 002$ & $0 \cdot 0003-0 \cdot 02$ & \\
Provinces & & & \\
$\quad$ Toledo & $0 \cdot 56$ & $0 \cdot 34-0 \cdot 92$ & $*$ \\
$\quad$ Cuenca & $0 \cdot 95$ & $0 \cdot 48-1 \cdot 87$ & \\
$\quad$ Guadalajara & $4 \cdot 15$ & $2 \cdot 50-6 \cdot 89$ & \\
$\quad$ Ciudad Real & $2 \cdot 30$ & $1 \cdot 49-3 \cdot 55$ & \\
Season of hospitalization & & & \\
$\quad$ (vs. Dec.-Feb.) & & & \\
$\quad$ March-May & $0 \cdot 50$ & $0 \cdot 36-0 \cdot 71$ & $*$ \\
$\quad$ June-August & $0 \cdot 08$ & $0 \cdot 05-0 \cdot 14$ & \\
$\quad$ September-November & $0 \cdot 37$ & $0 \cdot 28-0 \cdot 51$ & \\
Dehydration & $12 \cdot 44$ & $1 \cdot 52-40 \cdot 38$ & $0 \cdot 0003$ \\
Intravenous rehydration & $1 \cdot 74$ & $1 \cdot 29-2 \cdot 35$ & $0 \cdot 0020$ \\
Acidosis & $1 \cdot 51$ & $1 \cdot 24-1 \cdot 83$ & $*$ \\
Respiratory tract infections & $1 \cdot 60$ & $1 \cdot 09-1 \cdot 98$ & $0 \cdot 0033$ \\
Concomitant infectious AGE & $1 \cdot 52$ & $1 \cdot 03-2 \cdot 25$ & $0 \cdot 0355$ \\
\hline \hline
\end{tabular}

MBDS, Minimum Basic Data Set; CLM, Castile-La Mancha; OR, odds ratio; CI, confidence interval; AGE, acute gastroenteritis.

$* P<0 \cdot 0001$.

In the 3 years that Rotarix and RotaTeq were commercially available in Spain, the frequency of both ARGE and other non-specific codified viral enteritis occurring in children aged $<5$ years decreased by $14.5 \%$ and $18.9 \%$, respectively, in regard to the prevaccine period. Additional information on the prevalence of the AGE most commonly coded in hospital discharge records, classified by hospitalization period and age group, is provided in Supplementary Table S1. A more detailed analysis of the decrease in ARGE incidence rates throughout the vaccination period is explained elsewhere [16]. Despite that analysis revealing a significant decline in the incidence rate of ARGE in 2007-2009 compared to 2003-2005 (incidence rate ratio $0 \cdot 86,95 \% \mathrm{CI} 0 \cdot 78-0 \cdot 96$ ), in the present study no significant differences were found between the percentage of children with ARGE accompanied with PNPs, PDPE, or dehydration in 20072009 compared to 2003-2005 $(P=0 \cdot 260,0 \cdot 414$ and $0 \cdot 069$, respectively).

\section{DISCUSSION}

ARGE cases carry an increased risk of dehydration and metabolic acidosis, a greater need for IV rehydration, and higher comorbidity rates with RTIs and other AGE. While dehydration with acidosis occurred in a third of cases with a simultaneous RTI, both together were hardly observed in children with a concomitant malabsorption syndrome, and in no cases did they present with a concomitant infectious AGE.

Our results agreed with the need for IV rehydration that other authors have previously noted [17]. In Europe, the severity of infection accounts for $53 \%$ of cases compared to $31 \%$ for other cases of AGE [3]. Although there is no threshold of severity to indicate the need for hospitalization, rotavirus infections requiring admission are those assessed by clinicians as severe or at risk of becoming so. Consequently, the severity of an AGE correlates significantly with the incidence of hospitalization [18].

The proportion of enteropathogenic concomitant AGE in ARGE cases is reported to be $11 \%$, with Campylobacter spp. being the most frequent [19]. We estimated a lower frequency of concomitant AGE cases, with adenovirus as the most common cause of all co-infections; moreover, the proportion of concomitant Salmonella observed in our study was higher than that reported in the literature [19]. However, we found no significant increase in gastrointestinal complications in these cases, in contrast to what could reasonably be expected [20], probably due to a problem of underdiagnosis in the MBDS.

Functional gastroesophageal reflux is common during early infancy [21]. Since vomiting both characterizes ARGE and constitutes a side-effect of the rotavirus vaccine [22], we hypothesized that GERD could be contributing to the general severity of the infection and, in particular, to dehydration in the youngest infants. However, although $97 \%$ of GERD cases occurred in children aged $<2$ years, the entity was not significantly associated with ARGE in the multivariable analysis. As expected, complications of intussusception or necrotizing enterocolitis were not codified [23, 24].

Prematurity, LBW, and congenital pathologies are all associated with increased risk for ARGE hospitalization [25], but we could not specifically evaluate the role of premature birth because it was not coded in the MBDS. Instead, we computed the percentage of ARGE cases that were attributable to PNPs and DPSD and their implications. In fact, the percentage 
of premature birth and LBW in rotavirus hospitalizations calculated from the MBDS in Spain between 1999 and 2009 was as low as 0.08-0.84\% [26]. Hence, we presume relevant miss-codification from the MBDS when it is used as data source of risk factors for rotavirus.

Because of its neurotropism, rotavirus is the most important agent in benign seizures caused by an AGE [27, 28], with seizures occurring in 3\% of ARGE cases with no pre-existing neurological conditions [29]. In CLM, seizures were coded less often and only $1.3 \%$ of ARGE cases with seizures were complicated with dehydration. It is well known that early rotavirus infection in the first 2 years of life is associated with a higher risk for recurrent wheezing, although no further associations have been found with other atopic manifestations [29]. In this sense, we did not find atopic dermatitis to be significantly more frequent in ARGE cases.

One limitation of this study is that many aspects of clinical histories are either recorded unevenly or not included in coded diagnoses. Although fever is present in up to $79 \%$ of ARGE cases [2], we could not analyse it as a factor because its encoding rate was $<0.5 \%$. Similarly, we could not assess vomiting or LBW individually as a perinatal risk factor. Breastfeeding was likewise not coded in the MBDS. Under-coding of terms may explain the lack of statistical significance of certain characteristics evaluated in our study. Indeed, an important disagreement between the clinical data contained in the MBDS and the main diagnosis has been reported [23]. Regarding the ability of the MBDS to specifically identify ARGE cases, it has only been described for nosocomial infections [24].

To improve the detection of community-acquired rotaviruses from the MBDS, we examined the secondary diagnosis codes 008.61 accompanying undefined AGE as the principal diagnosis. Dehydration and hypovolaemic shock were not considered as they require multiple differential diagnoses with other entities. Nevertheless, their lack of inclusion as principal diagnostic codes accompanying the secondary code 008.61 may have led to a certain amount of misclassification [30]. As only $0 \cdot 3 \%$ of ARGE cases corresponded to elective admissions, the risk of bias due to nosocomial ARGE that might have been labelled as community-acquired should be minimal. Finally, some misclassifications are assumed to be inherent in the MBDS because of the nature of the coding process.

The main strength of our research is that most factors studied here had not been previously analysed either singly or in a multi-causal manner. We evaluated their interactions to correct for possible confounding that may have led to over- or under-estimation, or even misdirection when they had been analysed separately in previous studies. In this sense, PNPs, DPSD, GERD, seizures, and atopic conditions were all shown to be unassociated with rotavirus in the multivariate analysis. This was probably due to the confounding effect of age since these factors were coded most often in the youngest patients ( $70 \%$ in children aged $<7$ months).

A model based on administrative data led us to explore the joint influence of intertwined factors associated with the severity of ARGE. Age group and concomitant RTIs appear to contribute to the need both for IV rehydration and metabolite replacement to counteract metabolic acidosis. Although several of these conclusions only reinforce and validate what is already known, the SDFs included in the MBDS have proven to be an efficient tool for elucidating the clinical and epidemiological behaviour of infectious AGE cases, resembling the information obtained by chart review. The finding that adenovirus is the leading cause of concurrent AGE should be explored further because of its possible role in acute respiratory and gastrointestinal manifestations of ARGE.

Recent studies have demonstrated that risk-based immunization programmes are not optimum in terms of coverage for people at risk, proposing additional strategies to reduce the remaining morbidity [31]. Likewise, modern dynamic models of rotavirus transmission have assessed that universal immunization programmes would be the preferred option to bring additional herd immunity benefits for the prevention of severe ARGE cases [32, 33]. In this sense, our study provides further suggestive evidence for policymakers seeking a possible future universal vaccination.

\section{SUPPLEMENTARY MATERIAL}

For supplementary material accompanying this paper visit http://dx.doi.org/10.1017/S0950268816000881.

\section{DECLARATION OF INTEREST}

None.

\section{REFERENCES}

1. Huppertz HI, Salman N, Giaquinto C. Risk factors for severe rotavirus gastroenteritis. Pediatric Infectious Diseases Journal 2008; 27: S11-S19. 
2. Gimenez-Sánchez, et al. Rotascore Research Group. Multicenter prospective study analyzing the role of rotavirus on acute gastroenteritis in Spain. Acta Pediatrica 2010; 99: 738-742.

3. Forster $\mathbf{J}$, et al. Hospital-based surveillance to estimate the burden of rotavirus gastroenteritis among European children younger than 5 years of age. Pediatrics 2009; 123: e393-400.

4. Rinder M, et al. Burden of severe rotavirus disease leading to hospitalization assessed in a prospective cohort study in Sweden. Scandinavian Journal of Infectious Diseases 2014; 46: 249-302.

5. Wilking $\mathbf{H}$, et al. Ecological analysis of social risk factors for rotavirus infection in Berlin, Germany, 20072009. International Journal of Health Geographics 2012; 28: 11-37.

6. Salim H, et al. Risk factors of rotavirus diarrhea in hospitalized children in Sanglah Hospital, Denpasar: a prospective cohort study. BMC Gastroenterology 2014; 14: 54.

7. Medici MC, et al. Epidemiological aspects of human rotavirus infection in children hospitalized with acute gastroenteritis in an area of northern Italy. Acta Biomedica 2004; 75: 100-6.

8. Dennehy PH, et al. A case-control study to determine risk factors for hospitalization for rotavirus gastroenteritis in U.S. children. Pediatric Infectious Diseases Journal 2006; 25: 1123-1131.

9. Payne DC, et al. Active, population-based surveillance for severe rotavirus gastroenteritis in children in the United States. Pediatrics 2008; 122: 1235-1243.

10. Yang LM, Fang YC. Clinical investigate and epidemiological of rotavirus enteritis in children. Zhonghua Shi Yan He Lin Chuang Bing Du Xue Za Zhi 2011; 25: 371-373.

11. Zvizdić $\mathbf{S}$, et al. Clinical characteristics of rotaviruses disease. Bosnian Journal of Basic Medical Sciences 2004; 4: 22-24.

12. Paloniemi M, Lappalainen S, Vesikari T. Commonly circulating human coronaviruses do not have a significant role in the etiology of gastrointestinal infections in hospitalized children. Journal of Clinical Virology 2015; 62: 114-117.

13. Paloniemi M, et al. Human bocaviruses are commonly found in stools of hospitalized children without causal association to acute gastroenteritis. European Journal of Pediatrics 2014; 173: 1051-1057.

14. Zeng M, et al. Clinical relevance of human bocavirus with acute respiratory tract infection and diarrhea in children: a prospective case-control study. Zhonghua Er Ke Za Zhi 2010; 48: 580-584.

15. Mihalache D, et al. Clinical study of acute diarrhea caused by rotavirus. Revista medico-chirurgicală a Societắtii de Medici şi Naturalişti din Iaşi 2005; 109: 488-491.

16. Redondo O, Cano R, Simón L. Decline in Rotavirus Hospitalizations following the first three years of vaccination in Castile-La Mancha, Spain. Human Vaccines and Immunotherapy 2015; 11: 769-775.

17. Wildi-Runge $\mathbf{S}$, et al. A 4-year study on clinical characteristics of children hospitalized with rotavirus gastroenteritis. European Journal of Pediatrics 2009; 168: 1343-1348.
18. Freedman SB, Eltorky M, Gorelick M (Pediatric Emergency Research Canada Gastroenteritis Study Group). Evaluation of a gastroenteritis severity score for use in outpatient settings. Pediatrics 2010; 125: e1278-1285.

19. Lan W-T, et al. Concomitant rotavirus and Salmonella infections in children with acute diarrhea. Pediatrics and Neonatology 2009; 50: 8-12.

20. Valentini D, et al. Coinfection in acute gastroenteritis predicts a more severe clinical course in children. European Journal of Clinical Microbiology and Infectious Diseases 2013; 32: 909-915.

21. Singendonk MM, et al. Upper gastrointestinal motility: prenatal development and problems in infancy. Nature Reviews Gastroenterology and Hepatology 2014; 11: 545-555.

22. Therapeutic Goods Administration. Document of Rotateq ${ }^{\circledR}$ product information, September 2008 (http:// www.public.health.wa.gov.au/cproot/2220/2/RotaTeq $\%$ 20PI $\% 20 \% 28 \mathrm{Sep} \% 202008 \% 29$.pdf). Accessed 8 January 2015.

23. Calle JE, et al. Quality of the information contained in the minimum basic data set: results from an evaluation in eight hospitals. European Journal of Epidemiology 2000; 16: 1073-1080.

24. Redondo-González O. Validity and reliability of the minimum basic data set in estimating nosocomial acute gastroenteritis caused by rotavirus. Revista Española de Enfermedades Digestivas 2015; 107: 142-151.

25. Bruijning-Verhagen $\mathbf{P}$, et al. Targeted rotavirus vaccination of high-risk infants; a low cost and highly costeffective alternative to universal vaccination. $B M C$ Medicine 2013; 11: 112.

26. National Center of Epidemiology. Report on the situation of rotavirus infections in Spain. Years 1999 2009. Madrid: Carlos III Health Institute, 2010.

27. Lloyd MB, et al. Rotavirus gastroenteritis and seizures in young children. Pediatric Neurology 2010; 42: 404-408.

28. Ueda $\mathbf{H}$, et al. Clinical characteristics of seizures associated with viral gastroenteritis in children. Epilepsy Research 2015; 109: 146-154.

29. Reimerink J, et al. Early-life rotavirus and norovirus infections in relation to development of atopic manifestation in infants. Clinical and Experimental Allergy 2009; 39: 254-260.

30. Lindenauer PK, et al. Association of diagnostic coding with trends in hospitalizations and mortality of patients with pneumonia, 2003-2009. Journal of the American Medical Association 2012; 307: 1405-1413.

31. Cromer D, et al. The burden of influenza in England by age and clinical risk group: a statistical analysis to inform vaccine policy. Journal of Infection 2014; 68: 363-371

32. Atkins KE, et al. Impact of rotavirus vaccination on epidemiological dynamics in England and Wales. Vaccine 2012; 30: 552-564.

33. Shim E, et al. Impact of transmission dynamics on the cost-effectiveness of rotavirus vaccination. Vaccine 2009; 27: 4025-4030. 